\title{
Laparoscopy in small bowel obstruction - current status - review
}

\author{
Jacek Szeliga, Marek Jackowski \\ Department of General, Gastroenterological and Oncological Surgery, Collegium Medicum Nicolaus Copernicus University, Torun, Poland \\ Videosurgery Miniinv 2017; 12 (4): 455-460 \\ DOI: https://doi.org/10.5114/wiitm.2017.72330
}

\begin{abstract}
Introduction: Acute small bowel obstruction (SBO) is an urgent medical condition. Its diagnosis is based mainly on a clinical examination followed by confirmatory simple routine radiological examinations such as plain $X$-ray of the abdominal cavity or computed tomography (CT). However, a real surgical challenge is not a decision whether to perform a surgery, but a decision when and how to perform it.

Aim: To determine the place of laparoscopy in contemporary management of acute SBO based on the current literature. Material and methods: A review of the literature based on the Medline database and including mainly the period of 2013-2017 was performed.

Conclusions: With regard to SBO, laparoscopy is a technique showing its advantages resulting from a minimally invasive approach. However, SBO is still a condition where the use of laparoscopy is limited mainly to selected cases such as SBO caused by single adhesions or foreign bodies. A basic limitation of using this technique is advanced and complicated SBO and lack of sufficient technical skills of the surgeon.
\end{abstract}

Key words: laparoscopy, small bowel obstruction, acute abdomen.

\section{Introduction}

Classic causes of mechanical small bowel obstruction (SBO) are divided into external, internal and intramural. The first group is the most common, and its mechanism of action includes compression of the intestinal wall from the outside, resulting in intestinal obstruction, and it includes various intraperitoneal adhesions (60-75\%), cancer lesions (20\%), hernia (10-20\%, including internal hernia which is more and more common, especially after bariatric procedures - 1-2\%), intussusception and other (5\%). Internal causes of SBO include, among others, various foreign bodies: foreign bodies that have been swallowed, bile calculi, bezoars (2-4\%) or even parasites. Additionally, causes of impaired patency of the gastrointestinal tract may be associated with intestinal inflammation, intestinal wall fibrosis secondary to a trauma, bowel anastomotic stenoses, intestinal ischaemia or radiation therapy complications. These intramural causes are observed in approximately $5 \%$ of all cases of SBO [1-4].

Initial management in a patient diagnosed with intestinal obstruction is associated with rapid diagnostic and therapeutic management. It is not always associated with a need to perform an emergency surgical procedure. Routine management measures depend, to some extent, on possible causes of obstruction and include correction of fluid and electrolyte imbalance, management of pain and vomiting, gastric decompression and patient monitoring. A decision regarding a surgical intervention is made dynamically, considering the patient's general condition, the probable cause of obstruction, as well as the intensity and dynamics of its symptoms. In extreme cases, such as cases with coexisting ischaemia and secondary intestinal gangrene, a surgical

\section{Address for correspondence}

Jacek Szeliga MD, PhD, Department of General, Gastroenterological and Oncological Surgery, Collegium Medicum Nicolaus Copernicus University, 53-59 Św. Józefa St, 87-100 Torun, Poland, phone: +48 509207 072, e-mail: jacky2@wp.pl 
intervention introduced at the appropriate time may even affect the patient's survival.

When looking at epidemiological data, it seems obvious that the most common causes of interventions for SBO include fibrous adhesions, which are a pathogenetic consequence of impaired integrity of the peritoneum, inflammatory processes, and secondary imbalance between intraperitoneal repairs and fibrinolysis. Such disorders lead to damage of phospholipid barriers isolating individual peritoneal membranes between which adhesions are formed [5]. These processes are especially severe in younger patients (below 60 years) and patients in whom the peritoneal integrity was lost due to medical procedures or traumas [6].

A literature review regarding SBO unequivocally indicates that laparoscopy is a method that becomes an effective tool in the surgeon's hands if patients have been selected appropriately. Moreover, it offers all the advantages of a minimally invasive procedure (reduced surgical trauma).

\section{Aim}

To determine the place of laparoscopy in contemporary management of acute SBO based on the current literature.

\section{Material and methods}

A review of the literature based on the Medline database and including mainly the period of 20132017 was performed. The analysis included only publications presenting adult patients and such that provided relatively reliable clinical data regarding this problem.

\section{Results and discussion}

Acute SBO is an urgent medical condition observed in almost $16 \%$ of all acute surgical cases (USA), and it requires urgent medical decisions on how to proceed. So far, the literature includes discussions over superiority of surgical methods over conservative treatment and discussions over the opposite approach $[7,8]$.

It is relatively easy to diagnose this condition, as its diagnosis is based mainly on a clinical examination followed by confirmatory simple routine radiological examinations such as plain $\mathrm{X}$-ray of the abdominal cavity or computed tomography
(CT). This latter examination is especially important with regard to precise detection of causes of obstruction, especially other than postoperative adhesions or strangulation, and at the same time its sensitivity and specificity reach $90 \%$ [5, 9]. Computed tomography is also important when planning a delay of a surgical intervention [7]. A routine ultrasound examination that is often underestimated due to technical issues caused by excessive intestinal gases may be diagnostically helpful especially in cases where intestinal ischaemia is also observed [5].

However, the real surgical challenge is not the decision whether to perform surgery, but the decision when and how to perform it. If symptoms progress dynamically, even hours may decide on the patient's fate. When symptoms are less intense and subacute, some surgeons allow 3-5 days for conservative treatment, after which a final decision about a surgical intervention has to be made [10]. Based on studies, some predictive parameters that may help make a decision to qualify patients for surgical treatment have also been determined. They include older age, presence of ascites, significant retention of gastric contents decompressed by a nasogastric tube or mesenteric swelling visible on $\mathrm{CT}[7,11]$. One should remember that in all cases surgical treatment alone is associated with statistically better long-term results and a statistically lower rate of recurrence of adhesions compared to conservative treatment; in the latter case as many as $31-40 \%$ of patients will have another obstruction that will require surgical treatment the most frequently $[5,7,12]$. Despite such data, it is necessary to proceed with caution, especially in the elderly, when assessing eligibility for surgical intervention.

As intraperitoneal adhesions are predominant with regard to epidemiology, the majority of reports describing surgical treatment for SBO are dedicated to this condition. With regard to this condition, a traditional approach still includes access to the peritoneal cavity via laparotomy, and one forgets that such abdominal opening is associated with a higher risk of subsequent postoperative adhesions. It is estimated that up to $30 \%$ of patients receiving such treatment will be considered eligible for another surgical procedure due to disease recurrence $[4,13]$. On the other hand, based on the analyses of clinical material it was determined that when using a laparoscopy technique alone in various abdominal proce- 
dures the rate of adhesion complications is reduced by as much as $45 \%$ [14].

The remaining indications for an intervention, mentioned above, are usually subjects of "case reports" or retrospective analyses with very small groups of patients. So far, there are no objective randomised clinical trials that could unequivocally determine appropriate eligibility criteria for a laparoscopic or open intervention in the case of SBO. An ongoing randomized controlled trial comparing a laparoscopic and classic approach in acute adhesive SBO will end in 2018 [15].

The main reason why a video approach is relatively unpopular in the treatment of mild SBO, especially adhesive obstruction, is a traditional, mainly historical, approach to its indications. In acute conditions, such as acute appendicitis or cholecystitis, minimally invasive procedures are a gold standard. However, gastrointestinal obstruction was one of the first contraindications for laparoscopy, but with time it has been changed to a relative contraindication. This approach has been explained variously. Firstly, laparoscopic surgery in the field with a limited space due to loop distension exposed patients to a significantly higher risk of iatrogenic damage to the intestinal wall and all associated consequences, especially when a trauma went unnoticed during surgery $[16,17]$. Then, animal studies demonstrated that iatrogenic perforation of an intestinal loop with secondary emptying of its contents when high pressure due to pneumoperitoneum is present may favour greater dynamics of bacterial translocation into blood, combined with the most serious septic consequences for a patient [18]. Due to all these factors even experienced surgeons approach such procedures with great caution.

Despite this, the first reports regarding attempts to use laparoscopy in the treatment of adhesive SBO are from the 1980s/1990s, when a minimally invasive approach only started its revolution in surgery [19]. As early as in this period Buianov et al. described the efficacy of laparoscopy in the diagnosis of acute SBO, which reached $94 \%$ [20]. Until now, the literature includes almost only retrospective analyses of small groups of patients and case reports and single meta-analyses regarding the problem of applying laparoscopy in SBO, such as an analysis by Li et al. in a group of 334 patients [21].

An answer to the question about the contemporary role of laparoscopy in cases of obstruction re- gards three aspects. First of all, laparoscopy may be treated as a final diagnostic tool that not only looks for the disease background but also definitively determines further surgical management with a minimum risk of complications. Moreover, with laparoscopy it is possible to locate a source of disease precisely and to perform a minimal incision that is optimally located under visual monitoring - the laparoscopic assisted open approach. And finally, laparoscopy may be used as a definitive therapeutic method with all its commonly known advantages [3, 4, 22].

In cases where gastrointestinal obstruction is observed when an intestinal loop overhangs or is rotated over a single fibrous band that only has to be cut and possibly coagulated, laparoscopy seems to be an ideal indication. Additionally, in cases where the background of SBO is not serious, improvement of patient comfort in the postoperative period, including shortened hospitalisation time, reduced rate of postoperative complications, hernia and pain, and reduced period of paralytic ileus, seems to be vital when selecting a method. Therefore, these elements have become a basis for analyses available in the literature.

In their statistical analysis Hackenberg et al. compared lysis of adhesions causing SBO using an open and laparoscopic approach and found statistically significant differences in favour of a minimally invasive procedure with regard to hospitalisation time $(p=0.007)$ and the use of operating theatre time $(p=0.01)$. They did not observe any significant differences regarding the number and severity of postoperative complications, including the rate of iatrogenic intestinal injury, which is the most important. However, as selection of patients into both study groups under comparison was not perfect, the obtained results have to be interpreted with some reservation. The conclusions emphasise benefits of the video approach, but based on their extensive experience the authors also concluded that clinical manifestation, medical history (especially surgical procedures in the past) and comorbidities should be considered when determining eligibility for a specific surgical procedure, and laparoscopy shows its advantages only in a selected group of patients [17]. Similar results were obtained by Kelly et al. in their retrospective study on a group of 9617 patients operated on due to SBO, $14.9 \%$ of whom underwent laparoscopy; they concluded that laparoscopy was associated with a significantly shorter time of hos- 
pitalisation $(p<0.0001)$ and of a procedure alone $(p<0.0001)$. Moreover, in patients operated on with a minimally invasive procedure there was also a significantly lower rate of complications, including surgical wound complications $(\mathrm{OR}=0.7,95 \% \mathrm{Cl}$ : $0.58-0.85, p<0.0001 ; \mathrm{OR}=0.22,95 \% \mathrm{Cl}: 0.15-0.33$, $p<0.0001)$ [23]. Similar observations regarding a significantly lower rate of complications associated with a laparoscopic procedure of adhesion lysis were obtained by Saleh et al., who performed a retrospective analysis of 4616 patients diagnosed with SBO (including 919, namely $19.3 \%$ treated laparoscopically) and demonstrated that both the mortality rate and complications were significantly lower for a video approach ( $p<0.01)$ [24]. On the other hand, Byrne et al., who used a video approach to perform a procedure in $30.9 \%$ out of 269 patients with SBO, confirmed beneficial effects of a minimally invasive approach on the rate of postoperative complications, which was significantly lower ( $p=0.002$ ) [25]. Regarding such reports, it is worth emphasising that the rate of conversion to open surgery is significant, almost $39 \%$ in the latter group, and even $42 \%$ in the publication by Nordin and Freedman [26]. It may indicate a surgeon's reasonable approach to surgical management in this surgical field that is so difficult for laparoscopy. This field is bordered by significantly distended intestinal loops, and therefore a procedure requires the highest surgical skills. The wall of intestines affected by obstruction is extremely sensitive to even the smallest mechanical traumas, and therefore some authors consider that there is an additional higher risk of its damage when managing laparoscopic tools. However, not all reports confirm this. Nonetheless, the latest population analysis by Behman et al. including 8584 cases of SBO indicates that apart from growing popularity of a laparoscopic approach in the treatment of adhesive SBO (an increase from $4.3 \%$ to $14.3 \%$ of all surgical procedures in the period 2005-2014), there is also a higher rate of repair intestinal surgery due to damage of the intestinal wall by laparoscopic tools. It has been calculated that a video approach is associated with a 1.6-fold risk of such damage [27].

A possibility of using a minimally invasive approach in elderly patients, namely above the age of 70 years, is also an important argument confirming the advantages of laparoscopy. Also in such cases results of laparoscopic procedures for SBO were completely comparable to laparotomy with regard to postoperative complications [28].

Taking these data into account, one should consider why laparoscopy is not as successful in SBO as in other indications despite being more and more popular and common. There is no unanimous answer to this question. It may be associated with technical difficulties resulting from physical limitations of the surgical field by distended intestinal loops, as well as problems with introducing trocars into the abdominal cavity that is tightly filled with intestines (often with adhesions), and therefore a decision to use a minimally invasive approach is not made even by the best laparoscopic surgeons. It is also undoubtedly an argument of opponents of a laparoscopic approach with regard to such procedures. Taking into account observations, a conventional limit for distended intestinal loops was set at $4 \mathrm{~cm}$, as this value allows for relatively safe performance of surgery with minimally invasive techniques. Moreover, it is also not recommended to perform laparoscopy for SBO in cases when the patient is not haemodynamically stable, there is diffuse peritonitis, multiple intra-abdominal abscesses and in patients after multiple surgical interventions in the abdominal cavity, especially when they have been caused by adhesions. Furthermore, one should always remember about increased frailty and sensitivity of the intestinal walls affected by obstruction. Therefore some authors recommend performing intensive decompression of the gastrointestinal tract with a nasogastric tube prior to the procedure [4]. Relative contraindications to use of this technique in SBO include insufficient expertise regarding management of laparoscopic tools.

The above data regard cases of SBO caused by obstruction due to fibrous adhesions. There are also many reports, mainly case reports, showing the efficacy of laparoscopy as a diagnostic and therapeutic method in cases of SBO due to rare causes: bezoars, bile stones, fruit pits, foreign bodies ingested whole, inflammatory processes causing intestinal retraction, and many others. When considering eligibility for laparoscopy, the role of precise diagnostic tests (e.g. CT) before surgery is emphasised. As laparoscopic advanced techniques of intestinal suturing and resection are common and distension of intestinal loops in such conditions is relatively small, minimally invasive procedures are favoured and can 
show their natural advantages over conventional surgery [3, 29-31].

\section{Conclusions}

With regard to SBO, laparoscopy is a technique showing its advantages resulting from a minimally invasive approach, including a reduced rate of complications, shorter hospitalisation period or lower consumption of analgesics. However, despite the fact that it is so commonly used and technically advanced, SBO is still a condition where the use of laparoscopy is limited in everyday practice mainly to selected cases such as adhesive SBO caused by single adhesions or foreign bodies in the gastrointestinal tract. A basic limitation of using this technique is advanced and complicated SBO and lack of sufficient technical skills of the surgeon.

\section{Conflict of interest}

The authors declare no conflict of interest.

\section{References}

1. Miraflor E. Green A. Small bowel obstruction. In: Abernathy's Surgical Secrets. $6^{\text {th }}$ ed. Harken AH, Moore EE (eds). Mosby Elsevier 2009; 48: 215.

2. Razavianzadeh N, Foroutan B, Honarvar F, Forozeshfard M. Small bowel obstruction attributable to phytobezoar. Oxford Medical Case Reports 2016; 2016: omw092. doi:10.1093/omcr/ omw092.

3. Sheikh AB, Akhtar A, Nasrullah A, et al. Role of laparoscopy in the management of acute surgical abdomen secondary to Phytobezoars. Cureus 2017; 9: e1363.

4. Kayano H, Nomura E, Kuramoto T, et al. Two cases of laparoscopic diagnosis and treatment of intersigmoid hernia. Tokai J Exp Clin Med 2017; 42: 109-14.

5. Ergul E, Korukluoglu B. Peritoneal adhesions: facing the enemy. Int J Surg 2008; 6: 253-60.

6. Tortella BJ, Lavery RF, Chandrakantan A, et al. Incidence and risk factors for early small bowel obstruction after celiotomy for penetrating abdominal trauma. Am Surg 1995; 61: 956-8.

7. Suh SW, Choi YS. Laparoscopy for small bowel obstruction caused by single adhesive band. JSLS 2016; 20: e2016.00048.

8. Miron A, Giulea C, Nadragea M, Enciu O. The laparoscopic approach of small bowel obstruction: the experience of a primary center. Chirurgia (Bucur) 2016; 111: 126-30.

9. Obuz F, Terzi C, Sökmen S, et al. The efficacy of helical CT in the diagnosis of small bowel obstruction. Eur J Radiol 2003; 48: 299-304.

10. Jeong WK, Lim SB, Choi HS, Jeong SY. Conservative management of adhesive small bowel obstructions in patients previously operated on for primary colorectal cancer. J Gastrointest Surg 2008; 12: 926-32.
11. Komatsu I, Tokuda Y, Shimada G, et al. Development of a simple model for predicting need for surgery in patients who initially undergo conservative management for adhesive small bowel obstruction. Am J Surg 2010; 200: 215-23.

12. Maung AA, Johnson DC, Piper GL, et al. Evaluation and management of small-bowel obstruction: an Eastern Association for the Surgery of Trauma practice management guideline. J Trauma Acute Care Surg 2012; 73: S362-9.

13. Landercasper J, Cogbill TH, Merry WH, et al. Long-term outcome after hospitalization for small-bowel obstruction. Arch Surg 1993; 128: 765-70.

14. Ten Broek RP, Issa Y, van Santbrink EJ, et al. Burden of adhesions in abdominal and pelvic surgery: systematic review and metaanalysis. BMJ 2013; 347: f5588.

15. Sallinen V, Wikström H, Victorzon M, et al. Laparoscopic versus open adhesiolysis for small bowel obstruction - a multicenter, prospective, randomized, controlled trial. BMC Surg 2014; 14: 77.

16. O'Connor DB, Winter DC. The role of laparoscopy in the management of acute small-bowel obstruction: a review of over 2,000 cases. Surg Endosc 2012; 26: 12-7.

17. Hackenberg T, Mentula P, Leppäniemi A, Sallinen V. Laparoscopic versus open surgery for acute adhesive small-bowel obstruction: a propensity score-matched analysis. Scand J Surg 2017; 106: 28-33.

18. Bustos B, Gómez-Ferrer F, Balique JG, et al. Laparoscopy and septic dissemination caused by perioperative perforation of the occluded small bowel: an experimental study. Surg Laparosc Endosc 1997; 7: 228-31.

19. Clotteau JE, Premont M. [Occlusion by adhesions treated by celioscopic section]. Presse Med 1990; 19: 1196.

20. Buianov VM, Perminova GI, Sirotinskil VV, Sokolov AA. [Laparoscopy in acute intestinal obstruction]. Klin Khir 1989; 4: 36-8.

21. Li MZ, Lian L, Xiao L, et al. Laparoscopic versus open adhesiolysis in patients with adhesive small bowel obstruction: a systematic review and meta-analysis. Am J Surg 2012; 204: 779-86.

22. Giordano A, Alemanno G, Bergamini C, et al. The role of laparoscopy in the management of a diagnostic dilemma: jejunal ectopic pancreas developing into jejunojejunal intussusception. Case Rep Surg. 2017; 2017: 8452947.

23. Kelly KN, Iannuzzi JC, Rickles AS, et al. Laparotomy for small-bowel obstruction: first choice or last resort for adhesiolysis? A laparoscopic approach for small-bowel obstruction reduces 30-day complications. Surg Endosc 2014; 28: 65-73.

24. Saleh F, Ambrosini L, Jackson T, Okrainec A. Laparoscopic versus open surgical management of small bowel obstruction: an analysis of short-term outcomes. Surg Endosc 2014; 28: 2381-6.

25. Byrne J, Saleh F, Ambrosini L, et al. Laparoscopic versus open surgical management of adhesive small bowel obstruction: a comparison of outcomes. Surg Endosc 2015; 29: 2525-32.

26. Nordin A, Freedman J. Laparoscopic versus open surgical management of small bowel obstruction: an analysis of clinical outcomes. Surg Endosc 2016; 30: 4454-63.

27. Behman R, Nathens AB, Byrne JP, et al. Laparoscopic surgery for adhesive small bowel obstruction is associated with a higher risk of bowel injury: a population-based analysis of 8584 patients. Ann Surg 2017; 266: 489-98. 
28. Cocorullo G, Falco N, Tutino R, et al. Open versus laparoscopic approach in the treatment of abdominal emergencies in elderly population. G Chir 2016; 37: 108-12.

29. Al-Qallaf A, Shuaib A, Al-Sharaf K, Behbehani A. Acute appendicitis as a rare cause of mechanical small bowel obstruction case report. Qatar Med J 2017; 2017: 4.

30. Fernando AR, Bulathsinghela R, Samarasekera DN. An unusual cause of small bowel obstruction due to an ingested mango seed: a case report. BMC Res Notes 2017; 10: 549.

31. Cocorullo G, Tutino R, Falco N, et al. Laparoscopic ileocecal resection in acute and chronic presentations of Crohn's disease. A single center experience. G Chir 2017; 37: 220-3.

Received: 30.11.2017, accepted: 5.12.2017. 